\title{
Microbial Proteomics: Archaea to Cyanobacteria
}

\section{Young-Ho Chung}

Korea Division of Life Science, Korea Basic Science Institute, Daejeon 305-333 Korea

(chungyh@kbsi.re.kr)

Thermococcus onnurineus NA1, sulfur-reducing hyperthermophilic archaeon, is capable of $\mathrm{H}_{2}$-producing growth and considered to be hydrogenogenic carboxydotrophy. Utilization of formate as a sole energy source has been well studied in T. onnurineus NA1. However, whether formate could be used as its carbon source remains unanswered question. To obtain a global view on the metabolic characteristics in $\mathrm{H}_{2}$-producing growth, the quantitative proteome analysis of $T$. onnurineus NA1 grown on formate, $\mathrm{CO}$ and starch was performed by combining 1D-SDS PAGE with nano UPLC-MS ${ }^{\mathrm{E}}$ method. Our data propose that $\mathrm{CO}$ could be utilized as its sole energy source via electron transport mechanism to produce $\mathrm{H}_{2}$. Additionally, our data propose that $\mathrm{CO}_{2}$ produced from catabolic pathway or $\mathrm{CO}$ oxidation by $\mathrm{CODH}$ and $\mathrm{CO}-$ induced hydrogenase activities could subsequently be assimilated into the organic carbon. Overall, proteomic comparison of formate- and CO-grown cells with starch-grown cells revealed that one carbon compound such as formate and CO could be utilized as an efficient substrate to provide cellular carbon and/or energy in $T$. onnurineus NA1.

Unicellular Gloeobacter violaceus PCC 7421 is a Gram-negative photoautotrophic cyanobacterium. Gloeobacter violaceus PCC 7421 was divided at very early point of the phylogenetic tree and contained very primitive and unusual features like lack of thylakoidal membrane and missing of some photosystem components. In this study we performed proteomic analysis of Gloeobacter violaceus PCC 7421 through the following two approaches. First, we separate the total, soluble and insoluble fractions of Gloeobacter violaceus PCC 7421 and performed the two-dimensional electrophoresis (2-DE). Second, insoluble fractions were analyzed by FT-ICR mass spectrometer. After 2-DE separation in-gel digested spots were identified by the MALDI/TOF/TOF analysis about 115, 73 and 21 proteins were identified from total, soluble and insoluble fractions, respectively. Also insoluble fraction was digested using both chemical and tryptic in-solution digestion methods to obtain the more peptides with transmembrane regions. Following the FT-ICR analysis 785 proteins were identified and among them 139 proteins with 1 or more ( 14) transmembrane domains were analyzed using TMHMM program. Considering the number of theoretical proteins (965) with 1 TM about $14.4 \%$ of that proteins were found. Through these proteomic analyses we could determine the protein preparation methods and provide the 2-DE map of Gloeobacter violaceus PCC 7421. 